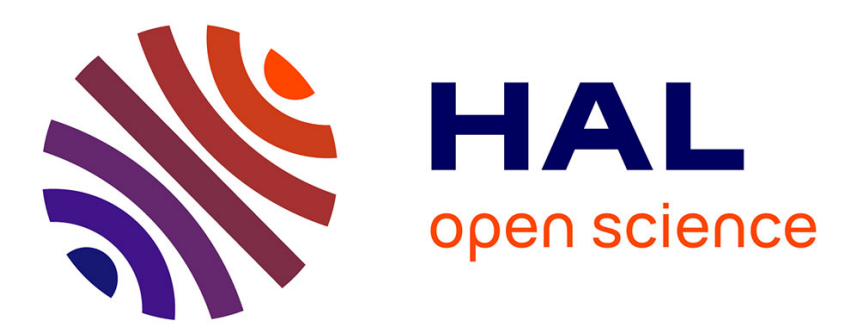

\title{
Photo-Induced Self-Assembly of Silver Nanoparticles for Rapid Generation of First and Second Surface Mirrors
}

Etienne Caillosse, Mohamed Zaier, Mariam Mezghani, Samar Hajjar-Garreau, Loïc Vidal, Daniel Lougnot, Balan Lavinia

\section{- To cite this version:}

Etienne Caillosse, Mohamed Zaier, Mariam Mezghani, Samar Hajjar-Garreau, Loïc Vidal, et al.. Photo-Induced Self-Assembly of Silver Nanoparticles for Rapid Generation of First and Second Surface Mirrors. ACS Applied Nano Materials, 2020, 3 (7), pp.6531-6540. 10.1021/acsanm.0c01010 . hal03014546

\author{
HAL Id: hal-03014546 \\ https://hal.science/hal-03014546
}

Submitted on 19 Nov 2020

HAL is a multi-disciplinary open access archive for the deposit and dissemination of scientific research documents, whether they are published or not. The documents may come from teaching and research institutions in France or abroad, or from public or private research centers.
L'archive ouverte pluridisciplinaire HAL, est destinée au dépôt et à la diffusion de documents scientifiques de niveau recherche, publiés ou non, émanant des établissements d'enseignement et de recherche français ou étrangers, des laboratoires publics ou privés. 


\section{Photo-Induced Self-Assembly of Silver}

\section{Nanoparticles for Rapid Generation of First and}

\section{Second Surface Mirrors}

Etienne Caillosse ${ }^{1,2}$, Mohamed Zaier $^{2}$, Mariam Mezghani ${ }^{2}$, Samar Hajjar-Garreau ${ }^{2}$, Loïc Vidal ${ }^{2}$, Daniel Lougnot ${ }^{3}$, Lavinia Balan ${ }^{1,2 *}$

${ }^{1}$ Université d'Orléans, Conditions Extrêmes Matériaux Haute Température et Irradiation CNRS UPR 3079, F-45000, Orléans, France

${ }^{2}$ Université de Haute Alsace, Institut de Science des Matériaux de Mulhouse CNRS UMR 7361, F-68100, Mulhouse, France

${ }^{3}$ Université de Haute Alsace, Centre de recherches sur les Economies, les Sociétés, les Arts et les Techniques CRESAT EA 3436, F-68100 Mulhouse, France

*Corresponding author: E-mail: lavinia.balan@cnrs-orleans.fr; Tel: +33(0)23825565

\section{Keywords:}

Silver mirrors, photo-induced synthesis, silver nanoparticles layers, reflective coating, nanostructured material. 


\section{ABSTRACT:}

Interesting mirror-like, highly reflective and conductive silver films have been successfully generated directly on a glass slide using a simple and fast photo-induced approach. A waterbased photosensitive formulation containing the silver precursor and a photo-generator of free radicals was used. It was applied like paint and exposed to UV light for a few seconds, in the absence of any additional reducing agents and without further treatment of any kind. This approach allowed ultrathin films of silver nanoparticles to be generated in situ (AgNPs) on the back side of the glass substrate. The particles are homogeneously auto-assembled onto the glass surface and the glass/silver diopter exhibits a high reflective character. With such an experimental arrangement, reflective surfaces are obtained: in contact with the glass slide, which is called the second surface mirror, and on the air side, which is called the first surface mirror. Quite interestingly, these reflective and conductive thin films exhibit strong natural adhesion on the glass substrate without the need for enhancing treatments (sensitization or activation) to improve the adhesion of the silver layer to the glass, as usual with silver glass mirrors. The influence of several chemical and photonic parameters has been examined in order to optimize the final optical and electrical properties of these silver films.

\section{- INTRODUCTION}

Nowadays, mirrors of all types are part of most people's daily lives, from smart coated windows and bathroom equipment, car rear-view mirrors, optical instruments (microscopes and telescopes) to laser reading systems such as compact disc players and barcode scanners, and so on. These mirrors are commonly referred to as front or second surface mirrors depending on the 
position of the reflective metal layer on the front or rear side of the glass substrate [1]. In fact, glass is an ideal material to be coated with a reflective metal top layer to create a mirror. It offers an optimal combination of thermal, mechanical and optical properties that provide protection against degradation factors and aggressive external conditions. In addition, it helps preserving the optical image and ensures the dimensional stability of the mirror even under severe conditions (high temperature, humidity, sun exposure) as long as it is not exposed to extreme environments [2].

In recent years, the use of metal nanoparticles (MNPs) in advanced applications has attracted considerable attention from the scientific community because of their tunable optical properties. They have also stimulated a major research effort due to a large number of new and potentially promising applications in a wide range of fields such as photonics, opto and microelectronics, SERS detection, solar concentrators/reflectors or light filters [3]. In order to tailor them to these new types of applications, a great deal of research has been devoted, in particular, to the synthesis and manipulation of metal nanoparticles with a view to generating nano- or microlayers on a variety of substrates. In addition, they are also finding an increasing place in the manufacture of mirrors for arts, design and architecture of buildings. For example, nanoparticles are being used to customize the appearance of buildings or to make their air-conditioning systems more efficient and less energy-intensive by deflecting heat during high summer months. To achieve this, characteristics such as reflectivity, thermal and electrical conductivity or the durability of thin metal deposits become priorities. Hence the interest of many researchers in the study and development of new synthesis approaches (physical, chemical, photochemical...) of metallic nanolayers on glass or other substrates. 
Chemical methods are the most commonly used to synthesize thin layers of silver or gold MNPs with a mirror-like appearance. Traditionally, the Tollens reaction (or other methods based on the same principle) is used for this purpose. By adding a small amount of a reducing sugar (i.e. glucose) to the Tollens reagent (a solution containing silver nitrate, sodium hydroxide and aqueous ammonia), silver is formed, which is deposited as a mirror-like coating inside the reaction vessel [4-7]. This method is still of current use in the manufacture of common household mirrors.

Lili He et al. used a chemical method in which a commercial aqueous suspension of citratecoated silver nanoparticles (AgNPs) was gently poured into a mediator vessel that contained a mixture of water and an immiscible non-polar solvent. At the water/solvent interface the AgNPs/citrate assemblies are destabilized and NPs spontaneously self-assembled to form nanothick scales of silver, which settled by gravity at the bottom of the container. They were then collected, air-dried and deposited on a slide to form a mirror [8]. Another chemical method consists of reducing metal ions with a Lewis base in an aprotic solvent. Thus $\mathrm{Ag}(+)$ can be reduced to $\operatorname{Ag}(0)$ by $\mathrm{F}(-)$ in a mixture of $\mathrm{MeCN} / \mathrm{DMSO}$ to form silver mirrors on the inner surface of the reaction vessels. However, this method is only valid in aprotic solvents and requires an additional washing step to remove excess reagents [9]. In any case, the various chemical approaches most often come up against problems related to the toxicity of the reagents, solvents or stabilizers used and the delicate handling and post-treatment of the by-products.

Among the physical methods used to produce MNPs films, the Physical Vapor Deposition (PVD) approach is one of the best known; it has the advantage of not requiring solvents, but it uses heavy machinery and involves significant energy consumption [10]. Ghidelli et al. used an 
ex-situ synthesis of gold nanoparticles by pulsed laser deposition (PLD) to develop nanostructured Au films with tailored optical properties in a pure Ar background gas [11]. However, this method requires severe deposition conditions such as high background pressures, high number of laser shots (500 shots) and high temperature of post-deposition annealing treatments up to $650^{\circ} \mathrm{C}$. Moreover, other complex physical methods such as ion sputtering, pulsed laser ablation, e-beam deposition, spark discharge generation and spray pyrolysis could be used for generating thin films of MNPs [12-15]. However, all of these physical methods require a rigorous control of many technical parameters (voltage, initial and working chamber pressure, spraying speed, rapid thermal annealing time (RTA), temperature, degree of supersaturation, etc.).

Recently, our research group has introduced a novel photo-induced synthesis approach that allows the creation of nascent AgNPs and coat or mark a variety of material surfaces with metal NPs. This UV photoreduction process has made it possible, to directly functionalize plastic food packaging or other substrates and has allowed effective control of the shapes and sizes of the generated NPs to obtain specific plasmonic silver layers or biocide coatings $[16,17]$.

Moreover, in previous works, we introduced an innovative and totally photo-chemical approach which allows the photo-crosslinking of a acrylate formulation and simultaneously the photo-reduction of the metal precursor; the ester functions of the polymer act also as a stabilizers for these NPs. Hence, the innovation lies in the specific nano-assembly of the NPs in the form of an in-depth concentration gradient in the acrylate polymer matrix that endows the as synthesized metal/polymers nanocomposite coating with valuable optical properties and a mirror-like appearance $[18,19]$. 
In the present paper, we introduce an innovative and greener alternative that breaks with everything we have done before. It still consists in using a photo-induced process but this time without any stabilizer or polymer matrix. The mirrors are made up solely of a submicrometrically thick metal layer of self-aligned silver NPs. For this purpose, the implementation of a one-step one-pot process and non-toxic reagents was a priority. Mirrors were obtained by depositing a water-based photosensitive formulation directly onto the substrate to be silvered and illuminating the sample for a few seconds with a standard continuous UV source. The as-synthesized AgNPs layers appear as highly reflective mirrors with effective electrical properties. To our knowledge, this photo-induced approach to obtaining first and second surface mirrors has never been reported in the literature.

The notion of first and second surface mirrors is purely conventional; it refers to the configuration of the mirror and its support in relation to the observer: first surface mirror when the reflecting surface is seen in front of the support by the observer and second surface mirror when it is seen through the support (transparent). Each of these types of mirrors has specific advantages that determine their fields of application. The great advantage of the process described in this publication is that it allows the manufacture of either second surface mirrors or specimens with two reflective surfaces (first surface + second surface mirrors) and this only depending on the thickness of the formulation and the photonic conditions of its implementation.

In addition, in order to obtain highly reflective layers with the lowest possible thickness (and thus save the maximum amount of silver precursor used), special attention has been paid to some particular aspects of the in situ generation of AgNPs, such as their spatial organization, homogeneity of their size and compactness of thin films. 


\section{- MATERIALS AND METHODS}

Materials. 2-hydroxy-2-methylpropiophenone used as a generator of primary free radicals was obtained from BASF. Silver nitrate $(\mathrm{AgNO} 3)$ purity $>99 \%$, diiodomethane and various solvents (ethanol, distillate water and acetonitrile) were from Sigma-Aldrich and used as received.

The samples were prepared on borosilicate microscope glass slides purchased from Roth. Before use, they have been treated to remove all traces of organic contamination. For this purpose, the $25 * 30 \mathrm{~mm}$ glass slides were soaked in sodium hydroxide solution N/10 (4 g/L) for about 10 minutes, then rinsed with abundant demineralized water and dried under air flow.

Instruments. Photochemical reactions were performed with a standard continuous UV LLC source from Heraeus Noblelight America equipped with an $\mathrm{H}$ bulb. This device provided a fluence of up to $2 \mathrm{~W} / \mathrm{cm}^{2}$ in the 200 to $600 \mathrm{~nm}$ window. Unless otherwise stated, the value used in this study was $800 \mathrm{~mW} / \mathrm{cm}^{2}$.

Reflectance measurements were carried out using a Thermo Fisher Scientific Evolution 200 UV-visible spectrophotometer with a $60 \mathrm{~mm}$ w/Spectralon ${ }^{\circledR}$ integrating sphere. The interrogating beam hit the sample under a $8^{\circ}$ incidence and a Teflon slide was used as a reference. In this configuration, the measured value was the total reflectivity, i.e. the sum of specular and diffuse reflection. In another configuration that uses another detector and bypasses the integrating sphere, this spectrophotometer was used to measure the transmittance of the samples. In general, reflectance and transmittance spectra are recorded between 300 and 1000 nm. 
Transmission electron microscopy (TEM) studies were carried out using a Jeol ARM-200F microscope (Tokyo, Japan) operating at $200 \mathrm{kV}$. The scanning electron microscopy images were obtained with an FEI Quanta 400 scanning electron microscope (SEM).

Atomic Force Microscopy (AFM) characterizations were performed on a FlexAFM Nanosurf microscope operating in tapping mode under ambient conditions using an ACT tip (AppNano), with a scanning frequency of $1 \mathrm{~Hz}$ and a resolution of $256 \times 256$ pixels. This device has been used to determine the thickness of silver films. For this, one calculates the difference in the altitude distribution along a scan perpendicular to a scratch made on the film by a hard tip. Height and roughness calculations were performed using the Gwydion freeware.

Samples were analyzed by X-ray photoelectron spectroscopy (XPS) on a VG Scienta (Uppsala, Sweden) SES 200-2 x-ray photoelectron spectrometer under ultra-high vacuum $(\mathrm{P}<10-9$ mbar). The monochromatized AlK $\alpha$ source $(1486.6 \mathrm{eV})$ was operated at a power of $420 \mathrm{~W}(30 \mathrm{~mA}$ and $14 \mathrm{kV}$ ). During acquisition, the pass energy was set to $100 \mathrm{eV}$ for high-resolution and $500 \mathrm{eV}$ for wide scan spectra. The deconvolution of the spectra into different components is performed with Gaussian (70\%) - Lorentzian (30\%) shaped peak or an asymmetry function in the case of Ag met using casaXPS software after having subtracted a Shirley-type background. The atomic percent is determine taking into account the transmission factor of the spectrometer, the inelastic mean free path, and the sensitivity factor of each atom.

\section{- RESULTS AND DISCUSSION}

Photo-induced synthesis of assembled NPs silvered mirror. The sample preparation protocol used in this study is similar with that used in our previous works [20, 21]. In a typical synthesis, about $10 \mathrm{mg}$ of 2-hydroxy-2-methylpropiophenone (ca $0.5 \%$ by weight) and about 
$100 \mathrm{mg}$ of silver nitrate (ca $5 \%$ by weight) were added to $2 \mathrm{~mL}$ of solvent and maintained for 60 minutes at room temperature under magnetic stirring to ensure complete dissolution. When the formulation was dropped onto the glass slide, the liquid film spontaneously spreads under the effect of its surface tension and low viscosity. However, in order to allow rapid and homogeneous and perfectly reproducible application of the formulation on the glass, an automatic bar coater was used for the deposition. For this, 4-5 drops (about $100-125 \mu \mathrm{L}$ ) of the photosensitive formulation were carefully deposited on the microscope slide placed horizontally and various bar coater were used ( $24 \mu \mathrm{m}, 12 \mu \mathrm{m}$ and $6 \mu \mathrm{m})$ to control the thickness of the liquid layer. The sample was then exposed to irradiation by the light source for a variable time (typ. 2040 seconds). Under these conditions, and with a $24 \mu \mathrm{m}$ bar coater, the optical density associated with the absorption of the free radical generator was about $0.02 @ 365$ nm, $0.07 @ 334 \mathrm{~nm}$ and $0.09 @ 313 \mathrm{~nm}$. Figure 1 illustrates the successive steps of the photo-induced process and the formation of thin silver layers on a glass substrate. Table 1 shows the range of solvents used for the preparation of sensitive formulations and the corresponding exposure times to UV light. The UV-exposure time is determined by the reactivity of each formulation and is therefore specific to each solvent or solvent mixture used. The irradiation is stopped when the surface of the film is totally dried (cured). In addition, exposure times have been optimized based on the evaluation of the properties of the samples and their stability over time. Too short an exposure time does not allow the sample surface to dry making it unstable. Conversely, too long an exposure time leads to a deterioration (whitening) of the metal layer, probably due in part, to bigger aggregates formed and some oxidation of the metal surface. 


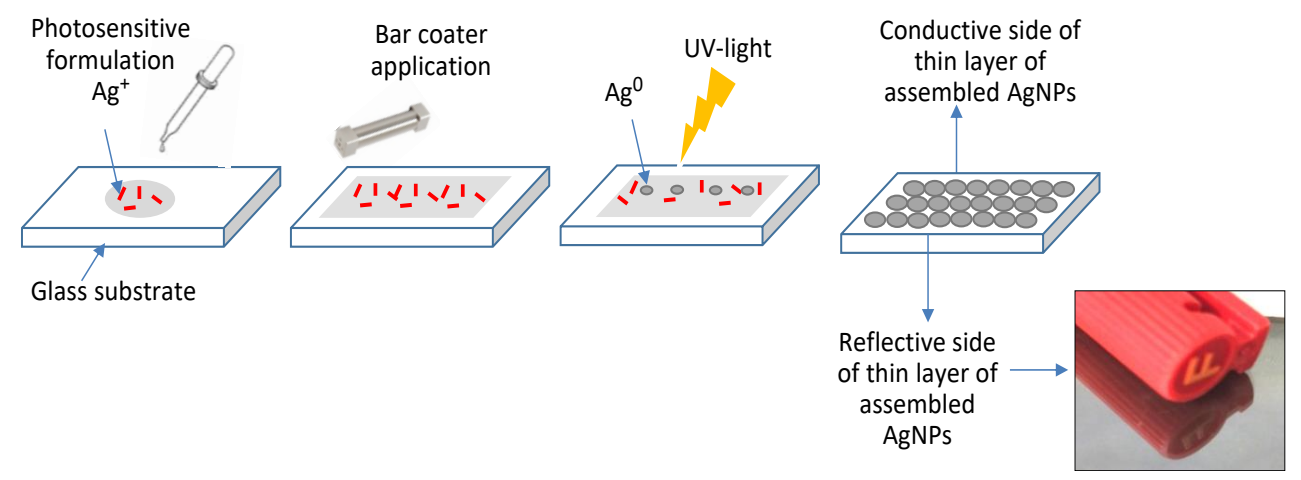

Figure 1. The successive steps of sample preparation and generation of the silver film by the photo-induced process.

Table 1. Photosensitive formulations with various solvents and the time exposure to UV light.

\begin{tabular}{cc}
\hline Solvents & $\begin{array}{c}\text { UV exposure } \\
\text { (sec) }\end{array}$ \\
\hline $100 \% \mathrm{H}_{2} \mathrm{O}$ & 40 \\
$100 \% \mathrm{C}_{2} \mathrm{H}_{5} \mathrm{OH}$ & 24 \\
$80 \% \mathrm{H}_{2} \mathrm{O}+20 \% \mathrm{C}_{2} \mathrm{H}_{5} \mathrm{OH}$ & 27 \\
$100 \% \mathrm{CH}_{3} \mathrm{CN}$ & 36 \\
$80 \% \mathrm{H}_{2} \mathrm{O}+20 \% \mathrm{CH}_{3} \mathrm{CN}$ & 32 \\
\hline
\end{tabular}

\section{Characterization of the AgNPs metalized layers.}

The structure of the layer as seen from the open air surface. The resulting silver coatings (air side) were analyzed by atomic force microscopy (AFM) to determine the topography and morphology of the nanoparticles forming the thin metal film. This characterization was performed in tapping mode. For each sample, a scan size of $2 \times 2 \mu \mathrm{m}$ was set with a resolution of $256 * 256$ pixels. Multiple scans, randomly located, were carried out on the surface of each sample, in order to scan it thoroughly. As can be seen in Figure 2, the spatial organization of the AgNPs remains uniform over the entire surface of the metal coating, with a roughness ranging 
from 7 to $100 \mathrm{~nm}$, depending on the thickness of the film. The thickness of the silver coating measured by the scratch technique is typically in the range of 50 to $350 \mathrm{~nm}$ (see Figure S1). The roughness parameter Ra corresponds to the arithmetic mean of the absolute deviations from the mean altitude calculated over a line drawn along the length of the sample [20]. This roughness has an impact on the reflective properties of the silver film on the air side. It can be observed that the roughness depends on the thickness of the sample. Interestingly, with the two thinner samples $(150$ and $50 \mathrm{~nm})$ this surface exhibits high reflectivity and is capable of forming the image of an object (see images Figure $2 b$ and $c)$.
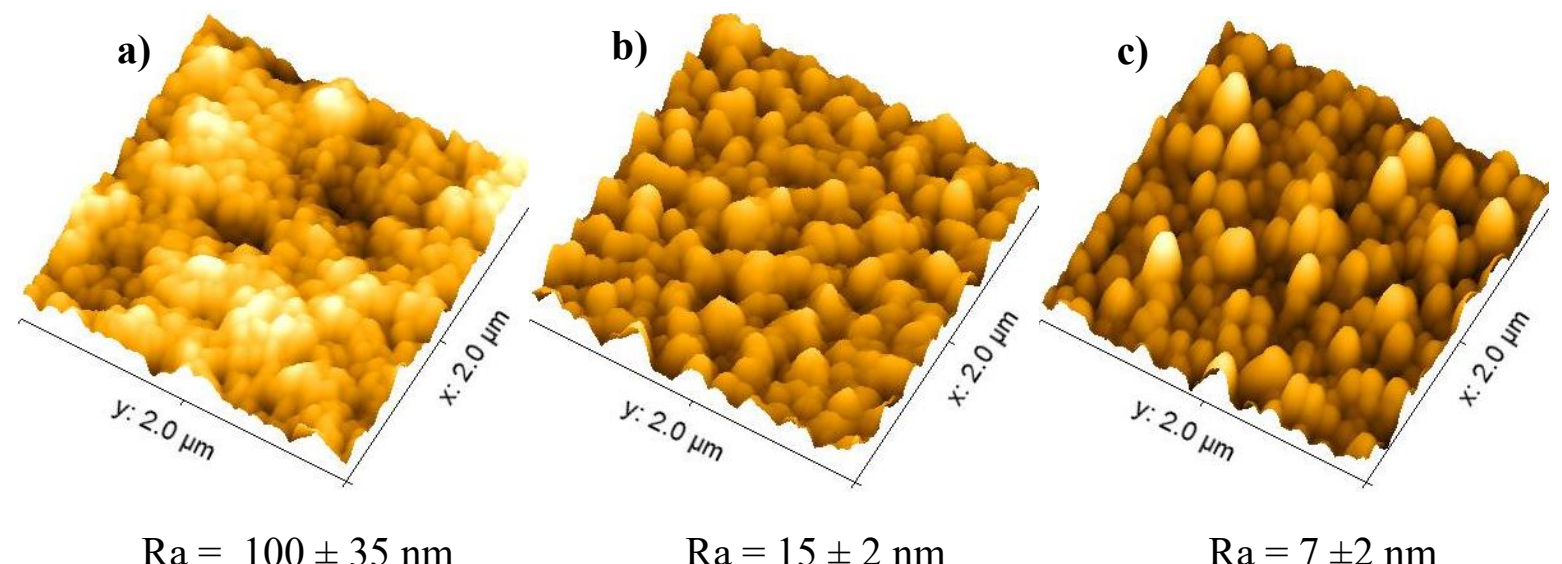

$$
\mathrm{Ra}=100 \pm 35 \mathrm{~nm}
$$
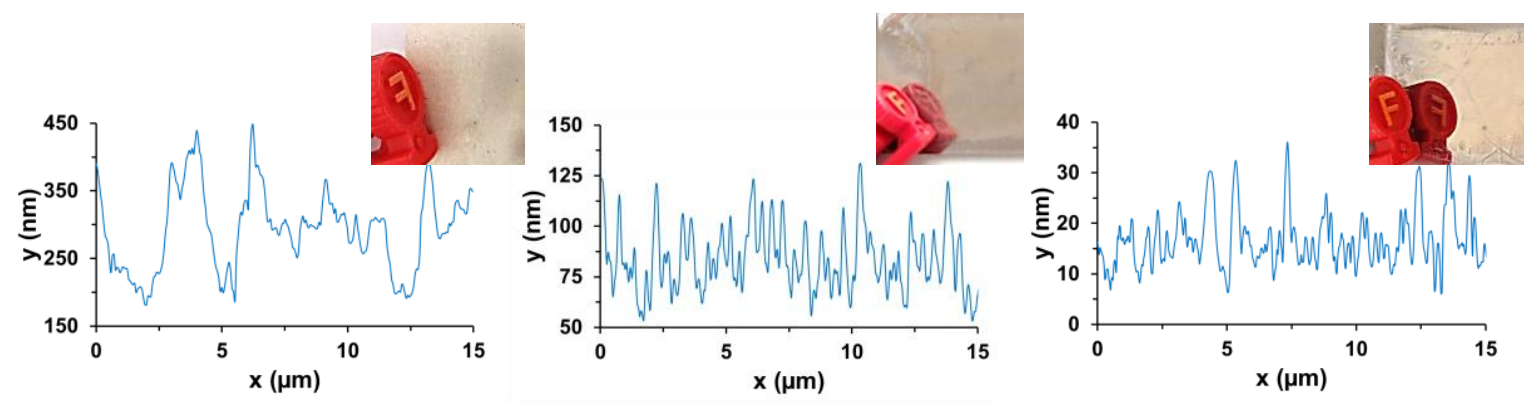

Figure 2. 3D AFM images of the air-side surface of $\mathrm{Ag}$ nanoparticle films of different thicknesses (acquired over $2 \times 2 \mu \mathrm{m}^{2}$ ) and roughness profile of each of the samples: a) Thickness $=350 \mathrm{~nm}, \mathrm{~b}) 150 \mathrm{~nm}$ and c) $50 \mathrm{~nm}(\mathrm{Ra}=$ arithmetic average roughness $)$, projections along the 
length of the sample and images of an object provided by these three silver films (Solvent: waterbased formulation, $\mathrm{UV}$ exposure $=40,25$ and $20 \mathrm{~s}$, bar coaters used 24,12 and $6 \mu \mathrm{m}$, respectively).

However, in the case of the thicker (and rougher) sample, the surface on the air side is only diffusely reflective and therefore cannot form an image (Figure 2a).

In order to perform analysis by transmission electron microscopy (TEM), a sample of the material of a thin reflective film of AgNPs was scrapped off from the glass substrate after synthesis. The recovered powder was dispersed in ethanol and a small drop was rapidly deposited on a TEM grid for analysis. As shown in Figures 3a and b, AgNPs exhibit a spherical shape with a diameter between 2 and $10 \mathrm{~nm}$. In addition, analysis of a batch of about $100 \mathrm{AgNPs}$ from a portion of the grid returned an average diameter of $5.8 \pm 0.5 \mathrm{~nm}$ (see insert in Figure 3a). The corresponding electron diffraction pattern of silver nanoparticles (boxed in the corner of Figure 3b) shows fringes corresponding to the 111, 200 and 220 planes of fec silver [21]. Figures $3 \mathrm{c}$ and $\mathrm{d}$ present the atomic resolution chemical mapping of a silver sample. This mapping is obtained by scanning energy dispersive $\mathrm{x}$-ray spectroscopy (SEDX). Figure 3c shows the nanoparticles against a background of carbon. The nanoparticles are well dispersed in the observed field with some agglomerates and a kind of spatial arrangement in a preferential direction seems to appear. Figure $3 \mathrm{~d}$ shows the elemental mapping of silver (in violet) which shows that the main element is silver. This result corresponds to the EDS analysis (Figure S2) which confirms the presence of silver as the very major element. We also observe in EDS analysis, some weak peaks of $\mathrm{O}$ and $\mathrm{C}$ which originate from the photolysis of the free radical generator and Si from the glass substrate (Figure S2). 

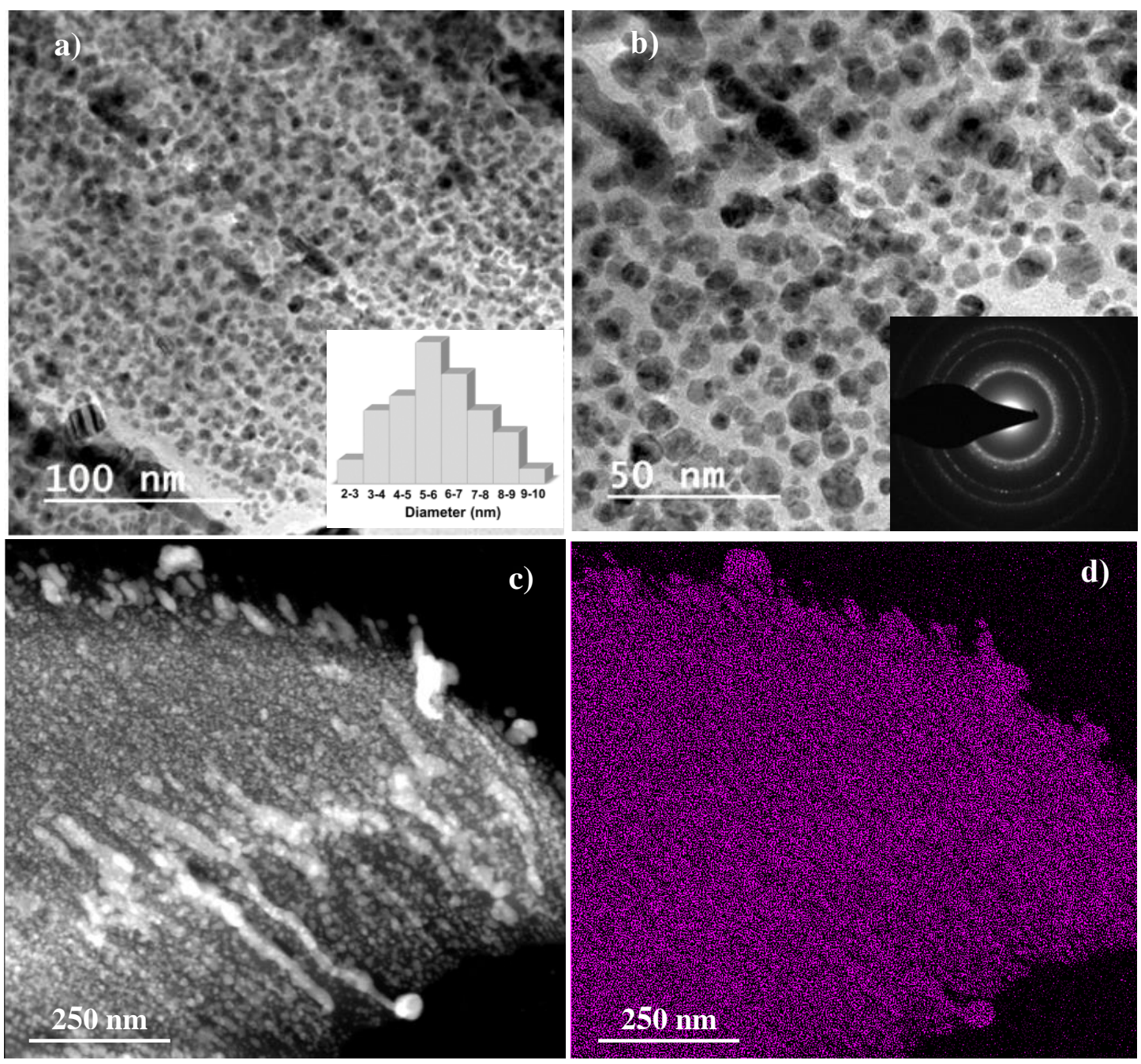

Figure 3. (Top) TEM images of the AgNPs film (thickness $=50 \mathrm{~nm}, 20 \mathrm{~s}$ UV exposure and water-based formulation); inset a) size dispersion of the particle and inset b) electron diffraction pattern. (Bottom) SEDX mapping with c) STEM image and d) silver elemental mapping (silver is figured in violet).

In addition, to learn more about the chemical composition of the metal layers, the open air surface of a thick film $(350 \mathrm{~nm}$, non-reflective on the air side and highly reflective on the glass side) was studied by X-ray photoelectron spectroscopy (XPS) Figure S3.The analysis shows a 
very intense binding energy double peak whose position corresponds to the spin-orbit splitting components $\mathrm{Ag} 3 \mathrm{~d}_{5 / 2}$ and $\mathrm{Ag} 3 \mathrm{~d}_{3 / 2}\left(\mathrm{Ag} 3 \mathrm{~d}_{5 / 2}\right.$ at $368.30 \mathrm{eV}$ and $\mathrm{Ag} 3 \mathrm{~d}_{3 / 2}$ at $374.30 \mathrm{eV}$ ) (see Figure S3). This confirms the presence of $\operatorname{Ag}(0)$ as the major constituent in the film layer [22]. No oxidized form of silver has been observed [23]. Signals of the elements C, O and H, which correspond to photoproducts resulting from the photolysis of the free radical generator present in the initial liquid formulation, were also present in the wide scan survey spectrum (Figure S3). A weak signal corresponding to traces of $\mathrm{N}$ in metal films was also detected (Figure S4). The results recorded with a series of mirror samples obtained from a variety of formulations are summarized in Table S1. They clearly confirm that $\operatorname{Ag}(0)$ is always the most predominant element at the surface of the sample, regardless of the solvent used to prepare the photosensitive formulation.

The reflectivity of the glass/silver diopter. Reflectivity was evaluated directly on the asprepared samples, without any further processing. According to the terminology adopted in this contribution, the mirror created at the glass/silver interface is designed as the second surface mirror. Its reflectivity was measured through the supporting glass plate between 300 and 1000 $\mathrm{nm}$ as shown in Figure 5 on thick samples (ca $350 \mathrm{~nm}$ ).

Usually, reflection spectrophotometers with a detection system based on an integrating sphere are designed to measure specular and diffuse reflection as a whole. As the equipment used operates in this way, a preliminary control was therefore made to ensure that in the samples studied, the contribution of diffuse reflection on the glass/silver diopter was negligible and thus the total reflection measured could be considered to correspond only to the specular component. For this purpose, the following experiment was carried out: the beam of a green $\mathrm{cw}$ laser $(\lambda=$ 
$532 \mathrm{~nm}$; beam diameter $=3 \mathrm{~mm}$; divergence $<1.2 \mathrm{mrad}$ ) was directed onto the reflecting surface under an incidence of $5^{\circ}$. The distribution of light energy in a plane perpendicular to the direction of propagation of the reflected beam was measured after a travel of 50 then $100 \mathrm{~cm}$ using a beam analyser. Comparison of the results obtained for the five samples described in Table 1 with that corresponding to the interrogating beam itself after an identical travel does not reveal any significant difference. This confirms that the contribution of diffuse reflection on the glass/silver diopter can be disregarded in this batch of samples. Another issue related to the optical characteristics of these second surface mirrors deserves a brief comment. In fact, Fresnel reflection on the glass/air diopter of the support plate is well known to decrease with increasing wavelength; this feature is described by the Cauchy relationship [24]. Combining it together with the Fresnel relationship makes it possible to evaluate this spurious reflectivity under any incidence. Thus, all recorded reflectance spectra have been corrected to take into account this spurious reflection, the values of which are reported in Table S2.

As can be seen from Figure 4 and Table S3, several formulations led to very interesting results. In particular, the three water-based systems have made it possible to generate second surface mirrors with a reflectance exceeding $80 \%$. In the case where the reaction medium was $\mathrm{H}_{2} \mathrm{O} / \mathrm{CH}_{3} \mathrm{CN}$, the reflectance was about $94 \%$, very close to that of optically polished solid silver [25]. Interestingly, the reflectance fluctuation in a batch of 5 supposedly identical replicas was measured to be $+/-5 \%$. On the other hand, systems formulated in acetonitrile or pure ethanol, which lead to mirrors with reflectance of $50-60 \%$, seem less promising. In an attempt to interpret these experimental observations, large differences in the saturated vapor pressure of these three solvents can be put forward. 

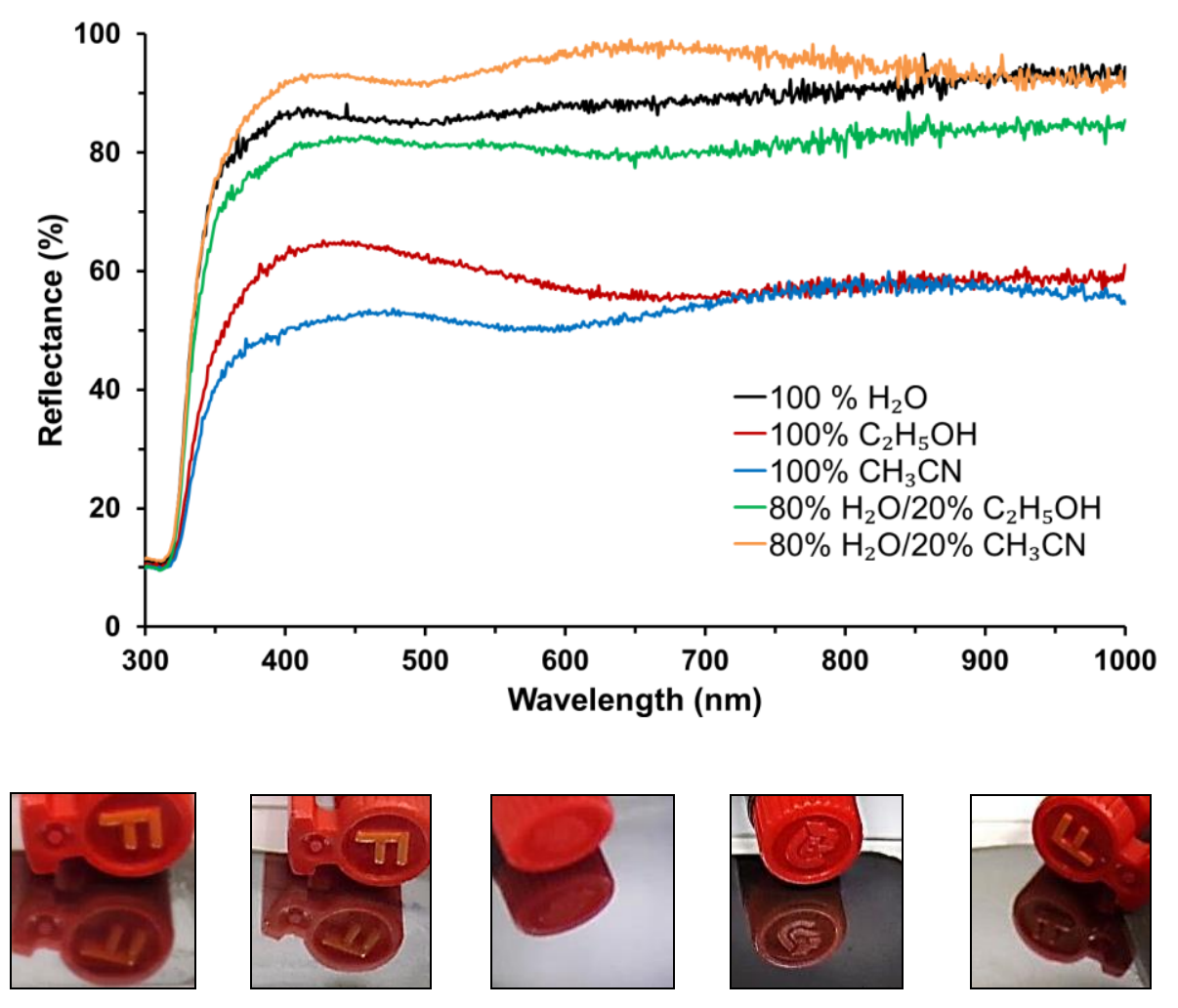

Figure 4. a) Reflectivity of the silver films on the glass side as a function of the solvents used for the photochemical reaction and $b$ ) corresponding pictures.

The saturated vapor pressures of ethanol $(6 \mathrm{kPa})$ and acetonitrile $(10 \mathrm{kPa})$ are about twice and four times that of water $(2.6 \mathrm{kPa})$, respectively. It is also important to keep in mind that the photo-induced process goes in line with a heating due both to the exothermicity of the photoreduction reaction of silver cations and most importantly, a degradation of the energy of the non-actinic photons into thermal energy. Thus, in parallel with the generation of native silver and the photoassisted creation of nanoparticles, the reaction medium evaporates. This phenomenon is therefore directly dependent on the saturated vapor pressure of the liquid phase. It could also be mentioned that the kinetic coupling between production of elementary nanoparticles and 
evaporation of the reaction medium is a key factor of the synthesis. While we have not yet rationalized this phenomenon, which is obviously a multi-parameter issue, the nature of the solvent has a decisive impact on the size and distribution of the NPs as already shown in our previous work [17] and at least two characteristics of the solvent are important: its polarity and its saturation vapour pressure. The polarity is involved in controlling the reduction reaction of the metal precursor and in the stabilization process of metal seeds and the saturation vapour pressure determines the rate of evaporation of the solvent. Moreover, rapid evaporation interrupts reduction prematurely and leads to an assembly of NPs not compact enough to achieve high reflectance. Conversely, slow evaporation promotes coalescence of particles and the formation of large aggregates, leading to a diffusing surface.

Modelling these phenomena will require a description of the thermal conditions of the experiment (exothermicity of the chemical reaction, heating due to plasmon resonance and deposition of parasitic heat by the actinic source) which is a very complex matter. It will be examined in a future study.

In addition, the coalescence process of NPs greatly influences the intimate structure and the compactness of the metal layer formed in this way. From this, it can therefore be inferred that these phenomena must also have an indirect influence on the electrical conductivity of the silver layers and on the quality of their adhesion to the glass substrate. Moreover, different concentrations of $\mathrm{Ag}^{+}$have been tested and 5\% is the optimized value of the silver concentration. When the concentration of $\mathrm{Ag}^{+}$is lower ( $2 \%$ for example), spaced NPs on the glass were obtained but no mirror aspect and when the concentration is higher, the mirrors are very inhomogeneous with a lot of aggregates. 
In order to analyze how the thickness of silver layers impacts the reflectivity of second surface mirrors, a thorough study was carried out by gradually reducing the quantity of the photosensitive formulation with water based. Figure 5 shows the reflection spectra of a set of three samples obtained under the same experimental conditions with water formulations leading to thicknesses of 50,150 and $350 \mathrm{~nm}$. The characteristic of these spectra that catches the attention is their overall shape: they approximately deduce from each other by a geometrical affinity (from one thickness to another, the reflectance is multiplied by a constant factor over the whole spectrum).

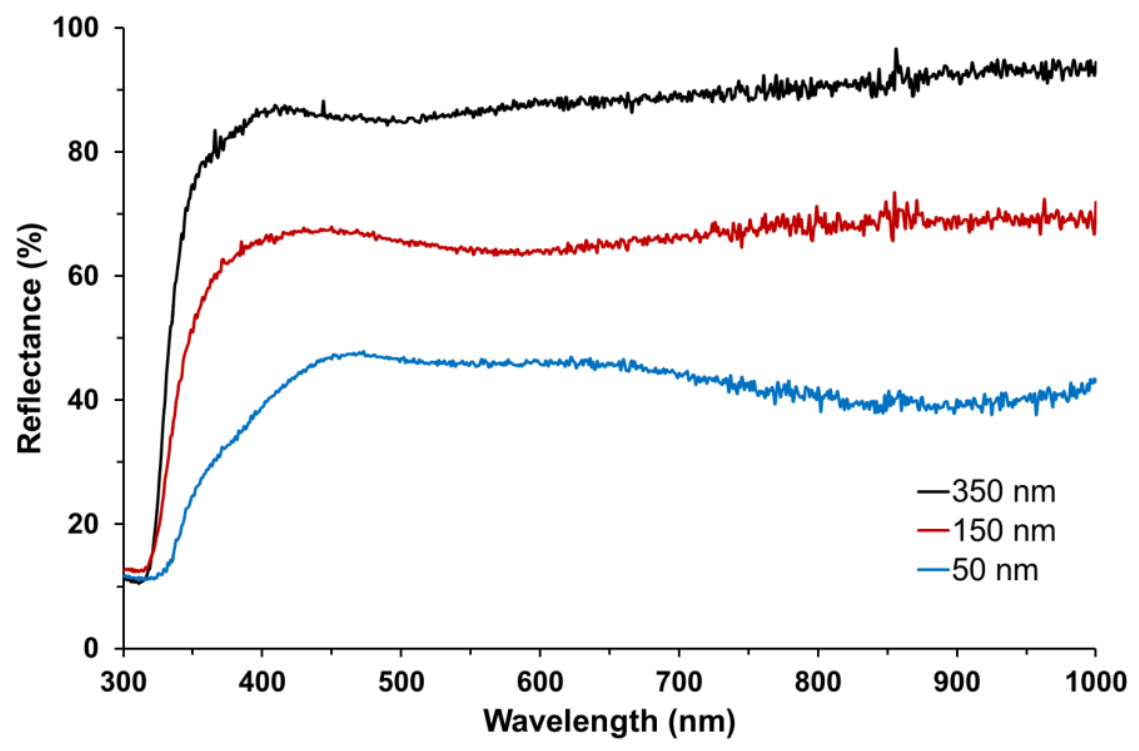

Figure 5. Reflectivity of silver mirrors on the glass side with increasing thickness $(50,150,350$ $\mathrm{nm}$ ) of the silver layer using a water-based formulation; bar coater 6,12 and $24 \mu \mathrm{m}$ and UV exposure time: 20,25 and $40 \mathrm{~s}$, respectively.

Such an observation suggests that what differentiates these three samples is the density i.e. the intimate structure of the metal layer. As thickness decreases, the stacking of nanoparticles 
becomes less compact so that the silver layer is no longer continuous. The evolution of the general shape of the reflection spectra as a function of the thickness of the layers is totally different from what has been reported by several research groups that have studied silver layers obtained by CPV (chemical vapor deposition) or have carried out theoretical calculations on this question [26]. Indeed, as the thickness decreases, the shape of the reflection spectrum is known to change. If the spectra deduce from each other by geometric affinity, it means that the observed effect is mostly due to the presence of more and more through-holes. The thickness of the layer certainly decreases a little, but at the same time it becomes more and more of a sort of strainer whose holes allow part of the incident light to pass through.

In order to further investigate the structure of semi-reflective layers - typically those with thicknesses in the order of $150 \mathrm{~nm}$ - the study of their properties was completed by recording their transmission spectrum (Figure 6). This makes it possible to draw up a balance of the light reflected ( $\mathrm{R} \%)$ and transmitted (T \%) by these samples. It can then be seen that there must be a third component since the sum of $\mathrm{R} \%+\mathrm{T} \%$ does not equal the totality of the incident light. When this deficit is reported as absorbance $[100-(\mathrm{R} \%+\mathrm{T} \%)]$, one recognizes the typical absorption associated with the surface plasmon of large silver particles - a broad, almost symmetrical and unstructured absorption band with a maximum around $585 \mathrm{~nm}$ (Figure 6).

Indeed, to explain the presence of this plasmon band, one must consider that the elementary particles that form the reflecting film are juxtaposed, in close contact, but only incompletely sintered and coalesced. As a result, the dielectric confinement at the origin of the plasmon resonance in isolated elementary nanoparticles is partly preserved. 


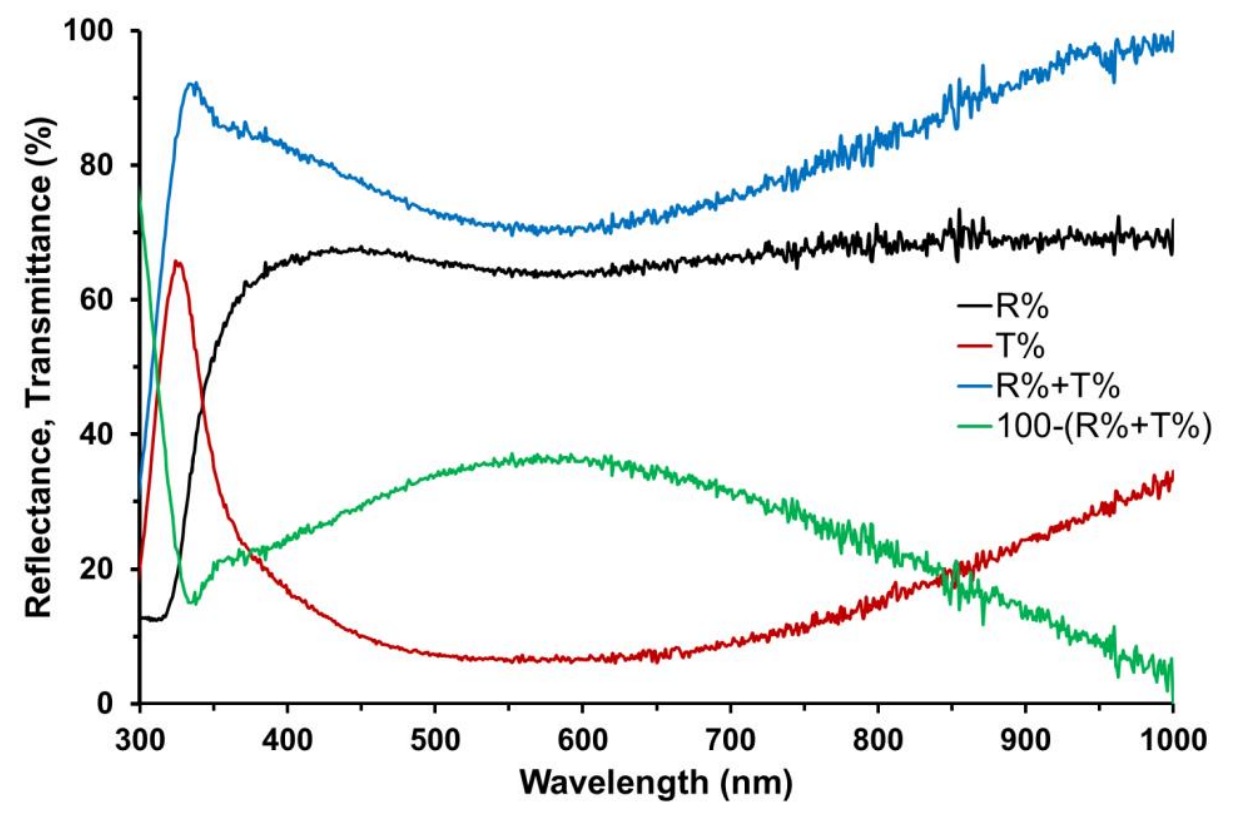

Figure 6. Reflection $(\mathrm{R} \%)$, transmission $(\mathrm{T} \%)$, reflection + transmission $(\mathrm{R} \%+\mathrm{T} \%)$ and complement to $100 \%(100-(\mathrm{R} \%+\mathrm{T} \%))$ spectra. (Solvent: water based formulation, thickness $=$ $150 \mathrm{~nm}, \mathrm{UV}$ exposure $=25 \mathrm{~s})$.

The reflectivity of the open air surface, i.e. first surface mirror. The AFM study of the surface topography on the open-air side revealed that for the thinnest layers (50 and $150 \mathrm{~nm}$ ), the measured rugosities were well below wavelength (see Figure 2). Therefore, it can be expected that the reflection occurring at these surfaces would be mainly of the specular type. Conversely, the reflectivity of thick samples, whose roughness is generally greater than $100 \mathrm{~nm}$, should be mainly diffuse reflection. Figure 7 presents the reflection spectra of the open-air surfaces of the thinner samples (50 and $150 \mathrm{~nm}$ ) studied by AFM along with illustrations showing the images of an object provided by these reflective surfaces (Figure 2). It was observed that the thicker the sample, the higher the reflectivity of the open air-surface (Figure 7). The air-surface roughness depend on the AgNPs size distribution and the gaps between the particles (see figure 3a) if are 
not filled with smaller ones, lowering in this way the reflectance [27]. However, as the thickness of the silver film increases, the roughness of the rear surface of the silver film also increases, to such an extent that the reflection from this surface ends up being only diffuse. This is effectively what happens with the thicker sample $(350 \mathrm{~nm})$, which for this reason, is no longer capable of forming a mirror image (see Figure 2a) [28].

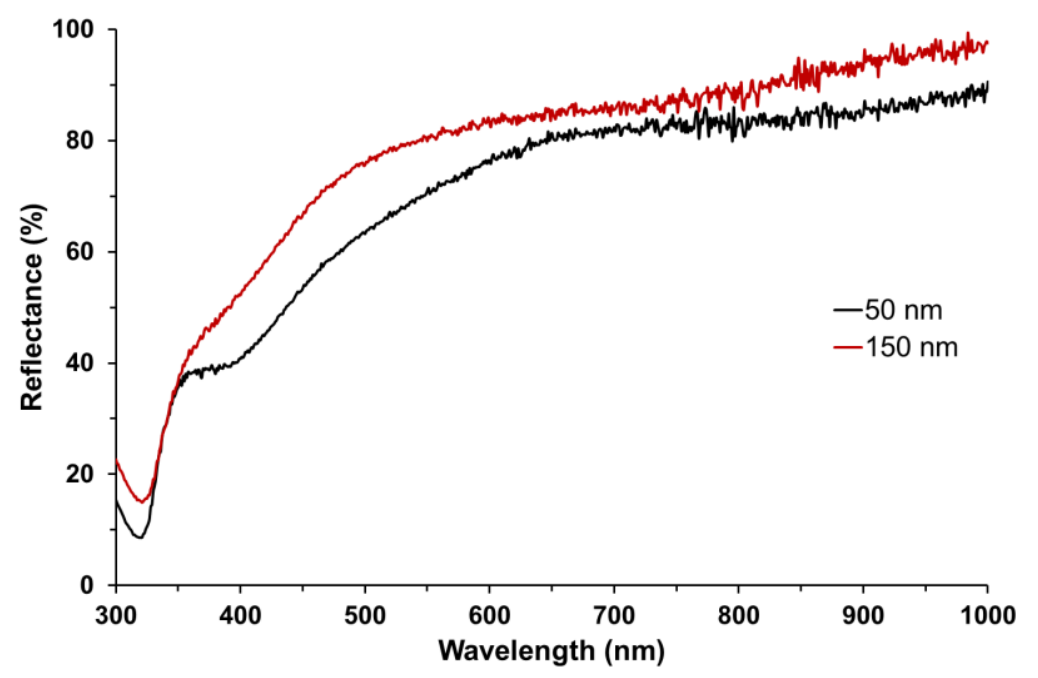

Figure 7. Reflectivity of the open air surface (rear surface) of the thinner silver mirrors (Solvent: water-based formulation, thickness $=150$ and $50 \mathrm{~nm}$, UV exposure $=25$ and $20 \mathrm{~s}$, respectively).

Electric resistivity of the silver layer. The resistivity of silvered layers was measured on the air-side with a two points Keithley 2000 device [29]. Regardless of the formulations and UV exposure times used, the general trend is that the various samples tested exhibit low resistivity. By way of illustration, resistivity data for $350 \mathrm{~nm}$ samples are collected in Table 2 . It is clear that this measuring approach provides only a very rough assessment of the conductivity of thin silver films. It does, however, reveal very large differences of about two orders of magnitude in the 
batch under consideration. Among the samples studied, with one exception, the higher the reflectivity, the better the electrical conductivity. This observation seems consistent with the notion of compactness of the layer formed by the assembly of AgNPs mentioned above to explain the differences in reflectivity observed in the same batch.

Table 2. Resistivity data of $350 \mathrm{~nm}$ silver coatings as a function of the solvents used to prepare the sensitive formulation.

\begin{tabular}{cc}
\hline Solvents & $\begin{array}{c}\text { Resistivity } \\
\text { (Ohm.cm) }\end{array}$ \\
\hline $100 \% \mathrm{H}_{2} \mathrm{O}$ & 10 \\
$100 \% \mathrm{C}_{2} \mathrm{H}_{5} \mathrm{OH}$ & 100 \\
$100 \% \mathrm{CH}_{3} \mathrm{CN}$ & 1.2 \\
$80 \% \mathrm{H}_{2} \mathrm{O}+20 \% \mathrm{C}_{2} \mathrm{H}_{5} \mathrm{OH}$ & 20 \\
$80 \% \mathrm{H}_{2} \mathrm{O}+20 \% \mathrm{CH}_{3} \mathrm{CN}$ & 4 \\
\hline
\end{tabular}

Nevertheless, the case of the sample prepared from a formulation based on acetonitrile is singular: it is at the same time, the least reflective and the most conductive. In any case, the conductivity property will have to be studied in greater depth using the much better adapted device known as the four-tip device.

Adhesion of the silver layer. Before considering possible practical applications of these second surface mirrors, it is necessary to ensure the quality of the adhesion of silver nano-layers on glass substrates. A test described by ASTM D3359 and ISO 2409 [30] is commonly used in the industrial world to evaluate the adhesion of coatings deposited on a variety of surfaces. It is known as the "Tape test". It consists in i) tracing a grid across the film to the substrate with a sharp razor blade, scalpel, knife or other cutting device, at a pitch of $1 \mathrm{~mm}$ with a spacing of 1 $\mathrm{mm}$, ii) applying a $25 \mathrm{~mm}$ wide, pressure sensitive tape onto the pattern (typ. with an adhesive 
force of $8.75 \mathrm{~N}$ ) and iii) peeling off the tape. The grid is then observed and the number of tiles torn off by the adhesive tape is recorded. Depending on the result, the adhesion is evaluated qualitatively on a scale of 0 to 5 . A score of 0 corresponds to the absence of chipping and 5 corresponds to the removal of more than $70 \%$ of the film by the tape. In our case, the adhesion evaluated on a set of about ten samples corresponds to a score of 0 , indicating excellent adhesion of silver nanolayers to glass (Figure S5). Although not yet clearly identified, the origin of this strong natural adhesion, two hypotheses can be put forward: i) most probably the first seeds are created on surface defects of the glass substrate which alone are capable of stabilizing them. And then the layer builds up from these hooking points; ii) photochemically generated primary radicals may also react with surface functions of the glass substrate or graft themselves onto this surface to create functions capable of strongly interacting with the silver seeds.

Wettability of the open air surface. In everyday applications of second surface mirrors (exterior, bathroom, car rear view mirror), it is necessary to protect the air side of the mirror against oxidation, corrosion or scratches. To do this, hard back coatings are generally applied to the AgNPs film. However, the choice of materials used in the formulation of these coatings is not obvious. The reason is that the wettability of this type of deeply corrugated surface at the submicrometer scale is often problematic. To access the apparent free energy of the air-side surface of silver mirrors, the static contact angles of water and diiodomethane on the surface of a sample with a silver layer thickness of ca $350 \mathrm{~nm}$ were determined. To do this, $2 \mu \mathrm{L}$ drops of deionized water and then diiodomethane were deposited on the sample surface, using a microsyringe and the contact angle was measured using a video capture device. To calculate the surface energy, Fowkes' mathematical model was used here [31]. 
Figure 8 shows a contact angle of approximately $110^{\circ}$ with water (Figure $8 \mathrm{a}$ ) (the deposited drop remains stable and does not flatten over time) and about $34^{\circ}$ with diiodomethane (Figure 8b). The calculated surface free energy is thus $43 \pm 2 \mathrm{mN} / \mathrm{m}$. This result is in line with the literature and is highly dependent on the roughness of the sample. Indeed, the more marked the surface roughness of a sample, the greater the contact angle with water. In the case under study, the Ra parameter was about $100 \mathrm{~nm}$ [32]. These results indicate that the $350 \mathrm{~nm}$ silver film behaves like a very hydrophobic material. The same experience was then repeated on the samples with much thinner silver films, which exhibit a lower roughness of their open air surface (see Figure 2). All the experimental results are gathered in Figure 8c and Figure S6.
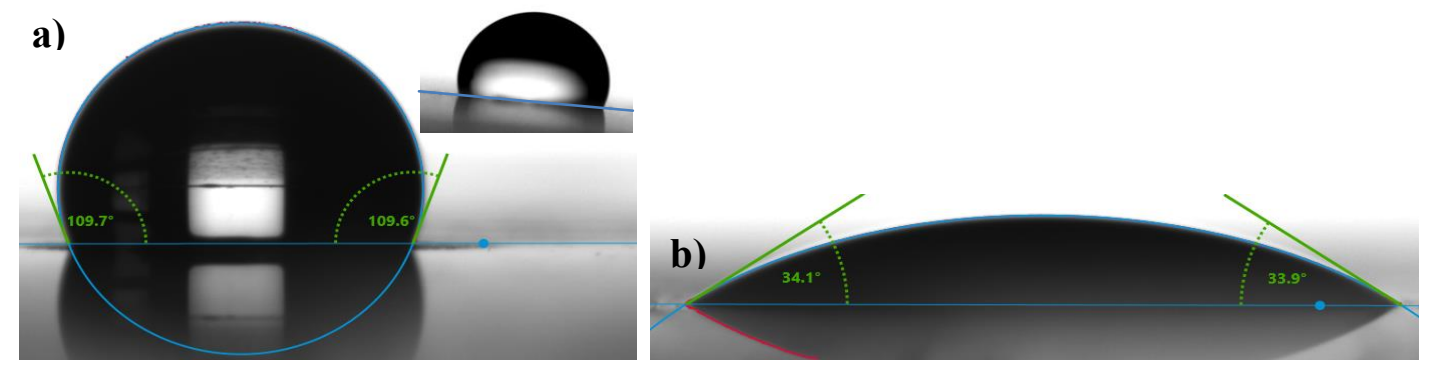

c) \begin{tabular}{ccc}
$\begin{array}{c}\text { Thickness } \\
(\mathbf{n m})\end{array}$ & $\begin{array}{c}\text { Roughness } \\
(\mathbf{n m})\end{array}$ & $\begin{array}{c}\text { Water contact } \\
\text { angle }\left(^{\circ}\right)\end{array}$ \\
\hline 350 & $100 \pm 35$ & $110 \pm 2$ \\
150 & $15 \pm 2$ & $91 \pm 3$ \\
50 & $7 \pm 2$ & $87 \pm 4$ \\
\hline
\end{tabular}

Figure 8. Contact angles on an water $350 \mathrm{~nm}$ thick silver film a) water drop (insert $20^{\circ}$ inclined support), b) diiodomethane drop and c) table with the contact angles of water as a function of the thickness and surface roughness of the three samples $(350,150$ and $50 \mathrm{~nm})$. 
Two models have been developed to explain the hydrophobicity of this type of highly uneven surface, the Cassie-Baxter model and the Wenzel model. The first considers that the roughness of the substrate and the small fraction of solid in contact with the liquid, combined with the micropockets of air trapped between the surface and the liquid, increase the contact angle. Wenzel's model, on the contrary, explains that the liquid fills all the porosity of the surface, due to the roughness, increasing the contact surface area significantly, thus increasing the actual hydrophobicity. A simple way to decide on which model applies to the systems studied in this work is to deposit a drop of water on the surface and then tilt the support at an angle of $20^{\circ}$. If the drop slips off the support, Cassie-Baxter's law applies to the system in question; otherwise Wenzel's model applies [33].

In all of the systems studied throughout this work, the Wenzel model seems to apply systematically (Figure 9 and Figure S6). The surface energies evaluated must allow the coating intended to protect the mirrors to be applied without having to carry out special treatments usually required (passivation or adhesion promoter for primer and finishing paints).

\section{- CONCLUSION}

This article reports on an original and very efficient photo-induced process for applying a silver mirror coating onto a glass substrate by using a simple water-based photosensitive formulation and UV light. After photo-induced synthesis, silver nanoparticles self-assembled to generate thin layers which can exhibit reflectivity up to $90 \%$ with excellent adhesion to the glass substrate and behave as electrically conductive coatings. The process is carried out at room temperature, in the open air and is completed within a few seconds. By playing with chemical and photonic parameters, some improvements in the optical reflectivity and electrical 
conductivity of silver nanolayers have been achieved. Several solvents or solvent mixtures (water, ethanol, acetonitrile) were also tested with which ultra-thin (50 and $150 \mathrm{~nm}$ ) first and second surface mirrors were obtained.

The use of this innovative approach for the manufacture of first and second surface mirrors should open the door to numerous industrial applications; some of the most booming areas include printing of micro-electric circuits, advanced light reflectors, solar cells, thermal insulation or smart windows. The approach offers an easy, inexpensive and environmentally friendly process to generate large scale functionalized surfaces with highly reflective and conductive properties.

\section{- ASSOCIATED CONTENT}

\section{Supporting information}

Supporting information is available: AFM characterizations, EDXS spectrum, XPS analysis, additional results of the reflectance, images of the "Tape Test", and images of the water drops on silver films.

\section{- AUTHOR INFORMATION}

Corresponding Author

*E-mail: lavinia.balan@cnrs-orleans.fr ORCID

Lavinia Balan: 0000-0003-0144-401X

Notes

The authors declare no competing financial interest.

\section{- ACKNOWLEDGMENTS}

The authors express their thanks to the Agence Nationale de la Recherche (ANR) for financial support under contract ANR-16-CE08-0032-01 and SATT Conectus project MIRROR. 


\section{- REFERENCES}

[1] Ennaceri, H.; Benyoussef, A.; Ennaoui, A.; Khaldoun, A. Optical Properties of Front and Second Surface Silver-Based and Molybdenum-Based Mirrors. International Journal of Engineering and Technology, 2016, 8(6), 410-413. DOI: 10.7763/IJET.2016.V8.923

[2] García-Segura, A.; Fernández-García, A.; Ariza, M. J.; Sutter, F.; Valenzuela, L. Durability studies of solar reflectors: A review. Renewable and Sustainable Energy Reviews, 2016, 62, 453-467. DOI: 10.1016/j.rser.2016.04.06

[3] Shenashen, M.; El-Safty, S.; Elshehy, E. Synthesis, Morphological Control, and Properties of Silver Nanoparticles in Potential Applications. Part. Part. Syst. Charact, 2014, 31, 293-316. DOI: $10.1002 / p p s c .201300181$

[4] Liebig, J. Uber die Produkte der Oxydation des Alkohols. Ann. Pharm., 1835, 14, 140. DOI: $10.1002 /$ andp. 18351121012

[5] Yin, Y.; Li, Z.-Y.; Zhong, Z.; Gates, B.; Xia, Y.; Venkateswaran, S. Synthesis and characterization of stable aqueous dispersions of silver nanoparticles through the Tollens process. J. Mater. Chem., 2002, 12(3), 522-527. DOI: 10.1039/B107469E

[6] Qu, L.; Dai, L. Novel Silver Nanostructures from Silver Mirror Reaction on Reactive Substrates. J. Phys. Chem. B, 2005, 109(29), 13985-13990. DOI: 10.1021/jp0515838 
[7] Wang, Q.; Du, C.; Kong, L.; Hu, H. High sensitivity fibre surface plasmon resonance sensor based on silver mirror reaction. Transactions of the Institute of Measurement and Control, 2018, 40(2), 462-468. DOI: DOI: 10.1177/0142331216660357

[8] Qu, Y.; Tan, C.; Zhang, Z.; He, L. A facile solvent mediated self-assembly silver nanoparticle mirror substrate for quantitatively improved surface enhanced Raman scattering. Analyst, 2017, 142, 4075-4082. DOI: 10.1039/C7AN00784A

[9] Maity, K.; Panda, D.; Lochner, E.; Saha, S. Fluoride-Induced Reduction of Ag(I) Leading to Formation of Silver Mirrors and Luminescent Ag Nanoparticles. J. Am. Chem. Soc, 2015, 137(8), 2812-2815. DOI: $10.1021 / \mathrm{ja} 512020 \mathrm{w}$

[10] Perrenot, P.; Pairis, S.; Bourgault, D.; Caillault, N. Sulphur corrosion effect on the electrical performance of silver films elaborated by physical vapor deposition. Vacuum, 2019, 163, 26-30. DOI: 10.1016/j.vacuum.2019.01.024

[11] Ghidelli, M.; Mascaretti, L.; Bricchi, B. R.; Zapelli, A.; Russo, V.; Casari, C. S.; Bassi, A. L. Engineering plasmonic nanostructured surfaces by pulsed laser deposition. Applied Surface Science, 2018, 434, 1064-1073. DOI: 10.1016/j.apsusc.2017.11.025

[12] Nakata, Y.; Muramoto, J.; Okada, T.; Maeda, M. Particle dynamics during nanoparticle synthesis by laser ablation in a background gas. J. Appl. Phys., 2002, 91(3). DOI: $10.1063 / 1.1424048$. 
[13] Weber, A. P.; Seipenbusch, M.; Kasper, G. Application of Aerosol Techniques to Study the Catalytic Formation of Methane on Gasborne Nickel Nanoparticles. J. Phys. Chem. A, 2001, 105, 8958-8963. DOI: 10.1021/jp0115594

[14] Fryauf, D. M.; Diaz Leon, J. J.; Phillips, A.C.; Kobayashi, N. P. Silver Film Surface Modification by Ion Bombardment Decreases Surface Plasmon Resonance Absorption. ACS Appl. Mater. Interfaces 2017, 9, 15841-15847. DOI: 10.1021/acsami.7b01603

[15] Kim, J. H.; Germer, T. A.; Mulholland, G. M.; Ehrman, S.H. Size monodisperse metal nanoparticles via hydrogen-free spray pyrolysis. Adv. Mater, 2002, 14(7). DOI: 10.1002/15214095(20020404)14:73.0.CO;2-P

[16] Mustatea, G.; Vidal, L.; Calinescu, I.; Dobre, A.; Ionescu, M.; Balan, L. A photochemical approach designed to improve the coating of nanoscale silver films onto food plastic wrappings intended to control bacterial hazards. J Nanopart Res, 2015, 17(46). DOI: 10.1007/s11051-0142828-X

[17] Zaier, M.; Vidal, L.; Hajjar-Garreau, S.; Bubendorff, J.-L.; Balan, L. Tuning the morphology of silver nanostructures photochemically coated on glass substrates: an effective approach to large-scale functional surfaces. Nanotechnology, 2017, 28, 105603. DOI: $10.1088 / 1361-6528 / 28 / 10 / 105603$.

[18] Zaier, M.; Vidal, L.; Hajjar-Garreau, S.; Balan, L. Generating Highly Reflective and Conductive Metal Layers through a Light-Assisted Synthesis and Assembling of Silver Nanoparticles in a Polymer Matrix. Scientific Reports 2017, 7 https://doi.org/10.1038/s41598-017-12617-8. 
[19] Schejn, A.; Ott, M.; Dabert, M.; Vidal, L.; Balan, L. Photo-Induced Design of Reflective Metallized Gold@polymer Coatings with Tuned Architecture. Materials \& Design 2018, 160, 74-83. https://doi.org/10.1016/j.matdes.2018.08.051.

[20] Gadelmawla, E. S.; Koura, M. M.; Maksoud, T. M. A.; Elewa, I. M.; Soliman, H. H. Roughness parameters. Journal of Materials Processing Technology, 2002, 123, 133-145. DOI: 10.1016/S0924-0136(02)00060-2

[21] Qu, L.; Shi, G.; Wu, X.; Fan, B. Facile Route to Silver Nanotubes. Adv. Mater., 2004, 16, 1200-1203. DOI: 10.1002/adma.200400044.

[22] Ferraria, A.M.; Carapeto, A.P.; Botelho do Rego, A.M. X-ray photoelectron spectroscopy: Silver salts revisited. Vacuum, 2012, 86, 1988-1991. DOI: 10.1016/j.vacuum.2012.05.031

[23] Rajasekharreddy, P.; Usha Rani, P; Sreedhar, B. Qualitative assessment of silver and gold nanoparticle synthesis in various plants: a photobiological approach. J. Nanopart. Res., 2010, 12, 1711-1721. DOI: 10.1007/s11051-010-9894-5

[24] Chartier, G. Manuel d'optique, Paris, Hermès, 1997 (ISBN 2-86601-634-3), p. 437

[25] Taneja, P.; Ayyub, P.; Chandra, R. Size dependence of the optical spectrum in nanocrystalline silver. Physical Review $B, \quad 2002, \quad 65, \quad 245412 . \quad$ DOI: 10.1103/PhysRevB.65.245412

[26] Domaradzki, J.; Kaczmarek, D.; Mazur, M.; Wojcieszak, D.; Halarewicz, J.; Glodek, S.; Domanowski, P. Investigations of optical and surface properties of Ag single thin film coating as 
semitransparent heat reflective mirror. Materials Science-Poland, 2016, 34(4). DOI: $10.1515 / \mathrm{msp}-2016-0102$

[27] Yen, Y.-T.; Lu, T.-Y.; Lee, Y.-C.; Yu, C.-C.; Tsai, Y.-C.; Tseng, Y.-C.; Chen, H.-L. Highly Reflective Liquid Mirrors: Exploring the Effects of Localized Surface Plasmon Resonance and the Arrangement of Nanoparticles on Metal Liquid-like Films. ACS Appl. Mater. Interfaces, 2014, 6, 4292-4300. DOI: 10.1021/am406048s

[28] Zheng, B.; Peng Wong, L.; Wu, L. Y. L.; Chen, Z. Identifying Key Factors towards Highly Reflective Silver Coatings. Advances in Materials Science and Engineering, 2017. DOI: $10.1155 / 2017 / 7686983$

[29] Khan, M. A. M.; Kumar, S.; Ahamed, M.; Alrokayan, S. A.; AlSalhi, M. S. Structural and thermal studies of silver nanoparticles and electrical transport study of their thin films. Nanoscale Research Letters, 2011, 6, 434. DOI: 10.1186/1556-276X-6-434.

[30] Zainab, R. Standard Test Methods for Measuring Adhesion by Tape Test 1. ASTM International Designation: D3359 - 09'2, 2010, 1-8. DOI: 10.1520/D3359-09E02.

[31] Mirzaeian, M.; Ogwu, A. A.; Jirandehi, H. F.; Aidarova, S.; Ospanova, Z.; Tsendzughul, N. Surface characteristics of silver oxide thin film electrodes for supercapacitor applications. Colloids and Surfaces A: Physicochem. Eng. Aspects, 2017, 519, 223-230. DOI: 10.1016/j.colsurfa.2016.04.026 
[32] Zhou, Y.; Li, M.; Su, B.; Lu, Q. Superhydrophobic surface created by the silver mirror reaction and its drag-reduction effect on water. J. Mater. Chem., 2009, 19, 3301-3306. DOI: $10.1039 / \mathrm{b} 819251 \mathrm{k}$

[33] Lafuma, A.; David Quere, D. Superhydrophobic states. Nature Materials, 2003, 2, 457460. DOI: $10.1038 / \mathrm{nmat} 924$ 